\title{
Positive Solutions for Resonant and Nonresonant Nonlinear Third-Order Multipoint Boundary Value Problems
}

\author{
Liu Yang, Chunfang Shen, and Dapeng Xie \\ Department of Mathematics, Hefei Normal University, Hefei, Anhui 230061, China \\ Correspondence should be addressed to Liu Yang; yliu722@163.com
}

Received 5 December 2012; Accepted 24 January 2013

Academic Editor: Chuanzhi Bai

Copyright ( 2013 Liu Yang et al. This is an open access article distributed under the Creative Commons Attribution License, which permits unrestricted use, distribution, and reproduction in any medium, provided the original work is properly cited.

Positive solutions for a kind of third-order multipoint boundary value problem under the nonresonant conditions and the resonant conditions are considered. In the nonresonant case, by using the Leggett-Williams fixed point theorem, the existence of at least three positive solutions is obtained. In the resonant case, by using the Leggett-Williams norm-type theorem due to O'Regan and Zima, the existence result of at least one positive solution is established. It is remarkable to point out that it is the first time that the positive solution is considered for the third-order boundary value problem at resonance. Some examples are given to demonstrate the main results of the paper.

\section{Introduction}

We consider the existence of positive solutions for third-order $m$-point boundary value problem:

$$
\begin{gathered}
x^{\prime \prime \prime}(t)+f(t, x(t))=0, \quad t \in[0,1], \\
x^{\prime \prime}(0)=0, \quad x^{\prime}(0)=0, \quad x(1)=\sum_{i=1}^{m-2} \beta_{i} x\left(\xi_{i}\right),
\end{gathered}
$$

where $0<\xi_{1}<\xi_{2}<\cdots<\xi_{m-2}<1,0 \leq \beta_{i} \leq 1, i=$ $1,2, \ldots, m-2, \sum_{i=1}^{m-2} \beta_{i} \leq 1$, and $f \in C([0,1] \times[0, \infty), R)$.

If condition $\sum_{i=1}^{m-2} \beta_{i}=1$ holds, the problem is called resonant boundary value problem or boundary value problem at resonance. Otherwise, the associated problem is called nonresonant boundary value problem. Here, the condition $\sum_{i=1}^{m-2} \beta_{i}=1$ is denoted by (1.2).

Third-order differential equations arise in a variety of different areas of applied mathematics and physics, as the deflection of a curved beam having a constant or varying cross section, three-layer beam, and so on [1]. In recent years, the existence of positive solutions for nonresonant twopoint or three-point boundary value problems (Bvp for short) for nonlinear third-order ordinary differential equations has been studied by several authors. For examples, Anderson [2] established the existence of at least three positive solutions to problem

$$
\begin{gathered}
-x^{\prime \prime \prime}(t)+f(x(t))=0, \quad t \in(0,1), \\
x(0)=x^{\prime}\left(t_{2}\right)=x^{\prime \prime}(1)=0,
\end{gathered}
$$

where $f: R \rightarrow[0,+\infty)$ is continuous and $1 / 2 \leq t_{2}<1$.

By using the well-known Guo-Krasnoselskii fixed point theorem [3], Palamides and Smyrlis [4] proved that there exists at least one positive solution for third-order three-point problem:

$$
\begin{gathered}
x^{\prime \prime \prime}(t)=a(t) f(t, x(t)), \quad t \in(0,1), \\
x^{\prime \prime}(\eta)=0, \quad x(0)=x(1)=0, \quad \eta \in(0,1) .
\end{gathered}
$$

For more existence results of positive solutions for boundary value problems of third-order ordinary differential equations, one can see [5-12] and references therein.

For resonant problem of second-order or higher-order differential equations, many existence results of solutions 
have been established, see [13-25]. In [25], the authors considered the problem

$$
\begin{gathered}
x^{\prime \prime \prime}(t)=f\left(t, x, x^{\prime}\right)+e(t), \quad t \in(0,1), \\
x^{\prime}(0)=0, \quad x(1)=\beta x(\eta), \quad x(0)=\sum_{i=1}^{m-2} \alpha_{i} x\left(\xi_{i}\right) .
\end{gathered}
$$

By using the Mawhin continuation theorem, the existence results of solutions are established under the resonant condition $\beta=1, \sum_{i=1}^{m-2} \alpha_{i}=1, \sum_{i=1}^{m-2} \alpha_{i} \xi_{i}^{2}=0$ and $\beta=1 / \eta$, $\sum_{i=1}^{m-2} \alpha_{i}=1, \sum_{i=1}^{m-2} \alpha_{i} \xi_{i}^{2}=0$, respectively.

It is well known that the problem of existence for positive solution to nonlinear Bvp is very difficult when the resonant case is considered. Only few works gave the approach in this area for first- and second-order differential equations [26-31]. To our best knowledge, no paper deal with the existence result of positive solution for resonant third-order boundary value problems. Motivated by the approach in [27-29], we study the existence of positive solution for problem (1) under the nonresonant condition $\sum_{i=1}^{m-2} \beta_{i}<1$ and resonant condition $\sum_{i=1}^{m-2} \beta_{i}=1$, respectively. By using the Leggett-Williams fixed point theorem and its generalization $[28,30]$, we establish the existence results of positive solutions. The results obtained in this paper are interesting because

(1) the results obtained in the nonresonant case are more general than those established before;

(2) it is the first time that the positive solution is considered for third-order Bvp at resonance.

The rest of the paper is organized as follows. Some definitions and lemmas are given in Section 2. In Section 3, we consider the nonresonant case for problem (1). In Section 4, we discuss the existence of positive solution for problem (1) with resonant condition (1.2). Finally, in Section 5, we give some examples to illustrate the main results of the paper.

\section{Background Definitions and Lemmas}

For the convenience of the reader, we present here the necessary definitions and two-fixed point theorems.

Let $X, Y$ be real Banach spaces. A nonempty convex closed set $C \subset X$ is said to be a cone provided that

(1) $a x \in C$, for all $x \in C, a \geq 0$,

(2) $x,-x \in C$ implies $x=0$.

Definition 1. The map $\psi$ is said to be a nonnegative continuous concave functional on $C$ provided that $\psi: C \rightarrow+\infty$ is continuous and

$$
\psi(t x+(1-t) y) \geq t \psi(x)+(1-t) \psi(y)
$$

for all $x, y \in C$ and $t \in[0,1]$.
Definition 2. Let $0<a<b$ be given and let $\psi$ be a nonnegative continuous concave functional on the cone $C$. Define the convex sets $C_{r}$ and $C(\psi, a, b)$ by

$$
\begin{gathered}
C_{r}=\{x \in C \mid\|x\|<r\}, \\
C(\psi, a, b)=\{x \in C \mid a \leq \psi(x),\|x\| \leq b\} .
\end{gathered}
$$

Lemma 3 (the Leggett-Williams fixed point theorem [32]). Let $T: \bar{C}_{r} \rightarrow \bar{C}_{r}$ be a completely continuous operator and let $\psi$ be a nonnegative continuous concave functional on $C$ such that $\psi(x) \leq\|x\|$ for all $x \in \bar{C}_{r}$. Suppose that there exist $0<a<b<d \leq c$ such that

$$
\begin{aligned}
& \left(\mathrm{H}_{1}\right)\{x \in C(\psi, b, d) \mid \psi(x)>b\} \neq \varnothing \text { and } \psi(T x)>b, \text { for } \\
& \quad x \in C(\psi, b, d), \\
& \left(\mathrm{H}_{2}\right)\|T x\|<a \text { for }\|x\| \leq a, \\
& \left(\mathrm{H}_{3}\right) \psi(T x)>b \text { for } x \in C(\psi, b, c) \text { with }\|T x\| \geq d .
\end{aligned}
$$

Then, $T$ has at least three-fixed points $x_{1}, x_{2}$, and $x_{3}$ such that

$$
\left\|x_{1}\right\|<a, \quad b<\psi\left(x_{2}\right), \quad\left\|x_{3}\right\|>a, \quad \psi\left(x_{3}\right)<b .
$$

Operator $L: \operatorname{dom} L \subset X \rightarrow Y$ is called $a$ Fredholm operator with index zero, that is, Im $L$ is closed and $\operatorname{dim} \operatorname{Ker} L=\operatorname{codim} \operatorname{Im} L<\infty$, which implies that there exist continuous projections $P: X \rightarrow X$ and $Q: Y \rightarrow Y$ such that $\operatorname{Im} P=\operatorname{Ker} L$ and $\operatorname{Ker} Q=\operatorname{Im} L$. Moreover, since $\operatorname{dim} \operatorname{Im} Q=$ codim $\operatorname{Im} L$, there exists an isomorphism $J: \operatorname{Im} Q \rightarrow \operatorname{Ker} L$. Denote by $L_{P}$, the restriction of $L$ to $\operatorname{Ker} P \cap \operatorname{dom} L$ to $\operatorname{Im} L$ and its inverse by $K_{P}$, so $K_{P}: \operatorname{Im} L \rightarrow \operatorname{Ker} P \cap \operatorname{dom} L$ and the coincidence equation $L x=N x$ is equivalent to

$$
x=(P+J Q N) x+K_{P}(I-Q) N x .
$$

Denote $\gamma: X \rightarrow C$ to be a retraction, that is, a continuous mapping such that $\gamma x=x$ for all $x \in C$ and

$$
\begin{gathered}
\Psi:=P+J Q N+K_{P}(I-Q) N, \\
\Psi_{\gamma}:=\Psi \circ \gamma .
\end{gathered}
$$

Lemma 4 (the Leggett-Williams norm-type theorem [27]). Let $C$ be a cone in $X$, and let $\Omega_{1}, \Omega_{2}$ be open bounded subsets of $X$ with $\bar{\Omega}_{1} \subset \Omega_{2}, C \cap\left(\bar{\Omega}_{2} \backslash \Omega_{1}\right) \neq \emptyset$. Assume that $L: \operatorname{dom} L \subset$ $X \rightarrow Y$ is a Fredholm operator of index zero and

(C1) QN : X $\rightarrow Y$ is continuous and bounded, $K_{P}(I-$ Q) $N: X \rightarrow X$ is compact on every bounded subset of $X$,

(C2) $L x \neq \lambda N x$ for all $x \in C \cap \partial \Omega_{2} \cap \operatorname{dom} L$ and $\lambda \in(0,1)$,

(C3) $\gamma$ maps subsets of $\bar{\Omega}_{2}$ into bounded subsets of $C$,

(C4) $d_{B}\left(\left.[I-(P+J Q N) \gamma]\right|_{\text {ker } L}, \operatorname{Ker} L \cap \Omega_{2}, 0\right) \neq 0$, where $d_{B}$ stands for the Brouwer degree,

(C5) there exists $u_{0} \in C \backslash\{0\}$ such that $\|x\| \leq \sigma\left(u_{0}\right)\|\Psi x\|$ for $x \in C\left(u_{0}\right) \cap \partial \Omega_{1}$, where $C\left(u_{0}\right)=\left\{x \in C: \mu u_{0} \leq x\right\}$ for some $\mu>0$ and $\sigma\left(u_{0}\right)$ such that $\left\|x+u_{0}\right\| \geq \sigma\left(u_{0}\right)\|x\|$ for every $x \in C$, 
(C6) $(P+J Q N) \gamma\left(\partial \Omega_{2}\right) \subset C$,

(C7) $\Psi_{\gamma}\left(\bar{\Omega}_{2} \backslash \Omega_{1}\right) \subset C$,

then the equation $L x=N x$ has a solution in the set $C \cap\left(\bar{\Omega}_{2} \backslash \Omega_{1}\right)$.

\section{Positive Solution for \\ the Nonresonant Problem}

In this section, we suppose that $f \in C([0,1] \times$ $[0,+\infty),[0,+\infty))$ and $\sum_{i=1}^{m-2} \beta_{i}<1$. We begin with some preliminary results. Consider the problem

$$
\begin{gathered}
x^{\prime \prime \prime}(t)+y(t)=0, \quad t \in[0,1] \\
x^{\prime \prime}(0)=0, \quad x^{\prime}(0)=0, \quad x(1)=\sum_{i=1}^{m-2} \beta_{i} x\left(\xi_{i}\right) .
\end{gathered}
$$

Lemma 5. Denote $\xi_{0}=0, \xi_{m-1}=1, \beta_{0}=\beta_{m-1}=0$, then for $y(t) \in C[0,1]$, problems (10) and (11) have the unique solution

$$
x(t)=\int_{0}^{1} G(t, s) \int_{0}^{s} y(\tau) d \tau d s
$$

where

$$
\begin{aligned}
G(t, s)= & \frac{1}{1-\sum_{k=0}^{m-1} \beta_{k}} \\
& \times \begin{cases}(1-s)+\sum_{k=i}^{m-1} \beta_{k}\left(s-\xi_{k}\right), & t \leq s, \\
(1-t)+\sum_{k=0}^{i-1} \beta_{k}(t-s)+\sum_{k=i}^{m-1} \beta_{k}\left(t-\xi_{k}\right), & t \geq s,\end{cases}
\end{aligned}
$$

for $\xi_{i-1}<s<\xi_{i}, i=1,2, \ldots, m-1$.

Proof. Integrating both sides of (10) and considering the boundary condition $x^{\prime \prime}(0)=0$, we have

$$
-x^{\prime \prime}(t)=\int_{0}^{t} y(s) d s
$$

Let $G(t, s)$ be the Green function of problem

$$
\begin{gathered}
-x^{\prime \prime}(t)=0, \\
x^{\prime}(0)=0, \quad x(1)=\sum_{i=0}^{m-1} \beta_{i} x\left(\xi_{i}\right) .
\end{gathered}
$$

From (15), we can suppose that

$$
G(t, s)= \begin{cases}A+B t & t \leq s, \quad \xi_{i-1}<s<\xi_{i} \\ C+D t & t \geq s, \quad \xi_{i-1}<s<\xi_{i}\end{cases}
$$

For the definition and properties of the Green function together with (16), we have

$$
\begin{gathered}
A+B s=C+D s, \\
B-D=1, \\
B=0,
\end{gathered}
$$$$
C+D=\sum_{k=0}^{i-1} \beta_{k}\left(A+B \xi_{k}\right)+\sum_{k=i}^{m-1} \beta_{k}\left(C+D \xi_{k}\right)
$$

Hence,

$$
\begin{gathered}
A=\frac{1-s+\sum_{k=i}^{m-1} \beta_{k}\left(s-\xi_{k}\right)}{1-\sum_{i=0}^{m-1} \beta_{i}}, \\
C=\frac{1-\sum_{k=0}^{i-1} \beta_{k} s-\sum_{k=i}^{m-1} \beta_{k} \xi_{k}}{1-\sum_{i=0}^{m-1} \beta_{i}}, \\
B=0, \quad D=-1 .
\end{gathered}
$$

Thus,

$$
\begin{aligned}
G(t, s)= & \frac{1}{1-\sum_{k=0}^{m-1} \beta_{k}} \\
& \times \begin{cases}(1-s)+\sum_{k=i}^{m-1} \beta_{k}\left(s-\xi_{k}\right), & t \leq s, \\
(1-t)+\sum_{k=0}^{i-1} \beta_{k}(t-s)+\sum_{k=i}^{m-1} \beta_{k}\left(t-\xi_{k}\right), & t \geq s,\end{cases}
\end{aligned}
$$

for $\xi_{i-1}<s<\xi_{i}, i=1,2, \ldots, m-1$.

Considering (14) together, we obtain that problems (10) and (11) have the unique solution

$$
x(t)=\int_{0}^{1} G(t, s) \int_{0}^{s} y(\tau) d \tau d s .
$$

Lemma 6. The function $G(t, s)$ established in Lemma 5 satisfies that $G(t, s) \geq 0, t, s \in[0,1]$.

Proof. For $\xi_{i-1} \leq s \leq \xi_{i}, i=1,2, \ldots, m-1$ and $t \leq s$,

$$
(1-s)+\sum_{k=i}^{m-1} \beta_{k}\left(s-\xi_{k}\right) \geq \sum_{k=i}^{m-1} \beta_{k}\left(1-\xi_{k}\right) \geq 0 .
$$

For $\xi_{i-1} \leq s \leq \xi_{i}, i=1,2, \ldots, m-1$ and $t \geq s$,

$$
\begin{aligned}
(1-t) & +\sum_{k=0}^{i-1} \beta_{k}(t-s)+\sum_{k=i}^{m-1} \beta_{k}\left(t-\xi_{k}\right) \\
\geq & \sum_{k=0}^{i-1} \beta_{k}(1-s)+\sum_{k=i}^{m-1} \beta_{k}\left(1-\xi_{k}\right) \geq 0 .
\end{aligned}
$$

These ensures that $G(t, s) \geq 0, t, s \in[0,1]$. 
Lemma 7. If $y \in C[0,1]$ and $y \geq 0$, then the unique solution $x$ of problems (10) and (11) satisfy

$$
\min _{0 \leq t \leq 1} x(t) \geq \gamma \max _{0 \leq t \leq 1} x(t),
$$

where $\gamma=\left(\sum_{i=1}^{m-2} \beta_{i}\left(1-\xi_{i}\right)\right) /\left(1-\sum_{i=1}^{m-2} \beta_{i} \xi_{i}\right)>0$ is a constant.

Proof. For $x^{\prime \prime \prime}(t)=-y(t) \leq 0, t \in[0,1]$, we get that $x^{\prime \prime}(t)$ is decreasing on $[0,1]$. Then, the condition $x^{\prime \prime}(0)=0$ ensures that have $x^{\prime \prime}(t) \leq 0, t \in(0,1)$. This together with $x^{\prime}(0)=$ $0 x(t)$ is concave and decreasing on $[0,1]$. Thus,

$$
\max _{0 \leq t \leq 1} x(t)=x(0), \quad \min _{0 \leq t \leq 1} x(t)=x(1) .
$$

From the concavity of $x(t)$, we have

$$
\xi_{i}(x(1)-x(0)) \leq x\left(\xi_{i}\right)-x(0) .
$$

Multiplying both sides with $\beta_{i}$ and considering $x(1)=$ $\sum_{i=1}^{m-2} \beta_{i} x\left(\xi_{i}\right)$, we have

$$
\left(1-\sum_{i=1}^{m-2} \beta_{i} \xi_{i}\right) x(1) \geq \sum_{i=1}^{m-2} \beta_{i}\left(1-\xi_{i}\right) x(0) .
$$

This completes the proof of Lemma 7.

Let Banach space $E=C[0,1]$ be endowed with the maximum norm. We define the cone $C \subset E$ by

$$
\begin{aligned}
C=\{x \in E \mid & x(t) \geq 0, x^{\prime \prime}(0)=0, x^{\prime}(0)=0, \\
x(1) & \left.=\sum_{i=1}^{m-2} \beta_{i} x\left(\xi_{i}\right), x(t) \text { is concave on }[0,1]\right\} .
\end{aligned}
$$

Define the nonnegative continuous concave functional $\psi: C \rightarrow[0, \infty)$ by

$$
\psi(x)=\min _{0 \leq t \leq 1} x(t), \quad x \in C .
$$

Define the constants $\sigma, \delta$ by

$$
\sigma=\frac{1}{\max _{0 \leq t \leq 1} \int_{0}^{1} s G(t, s) d s}, \quad \delta=\int_{0}^{1} s G(1, s) d s .
$$

Theorem 8. Suppose that there exist constants $0<a<b<$ $b / \gamma \leq c$ such that
(A1) $f(t, x)<\sigma a,(t, x) \in[0,1] \times[0, a]$,
(A2) $f(t, x)>b / \delta,(t, x) \in[0,1] \times[b,(b / \gamma)]$,
(A3) $f(t, x)<\sigma c,(t, x) \in[0,1] \times[0, c]$,

then problem (1) has at least three positive solutions $x_{1}, x_{2}$, and $x_{3}$ satisfying

$$
\begin{gathered}
\left\|x_{1}\right\| \leq a, \quad b<\min _{0 \leq t \leq 1} x_{2}, \\
\left\|x_{3}\right\|>a \quad \text { with } \min _{0 \leq t \leq 1} x_{3}<b .
\end{gathered}
$$

Proof. We define operator $T: C \rightarrow E$ by

$$
T x(t)=\int_{0}^{1} G(t, s) \int_{0}^{s} f(\tau, x(\tau)) d \tau d s .
$$

It is easy to check that $T: C \rightarrow C$ and is completely continuous.

Next, the conditions of Lemma 3 are checked. If $x \in \bar{C}_{c}$, then $\|x\| \leq c$, and condition (A3) implies that

$$
\begin{gathered}
f(t, x) \leq \sigma c, \quad 0 \leq t \leq 1, \\
\|T(x)\|=\max _{0 \leq t \leq 1} \int_{0}^{1} G(t, s) \int_{0}^{s} f(\tau, x(\tau)) d \tau d s \\
\leq \max _{0 \leq t \leq 1} \int_{0}^{1} s G(t, s) d s \times \sigma c \leq c .
\end{gathered}
$$

Thus, $T: \bar{C}_{c} \rightarrow \bar{C}_{c}$. In the same way, we see that $T: \bar{C}_{a} \rightarrow$ $\bar{C}_{a}$. Hence, condition $\left(\mathrm{H}_{2}\right)$ of Lemma 3 is satisfied.

The fact that constant function $x(t)=(b / \gamma) \in$ $\{C(\psi, b,(b / \gamma)) \mid \psi(x)>b\}$ implies that $\{C(\psi, b,(b / \gamma))$ | $\psi(x)>b\} \neq \varnothing$. If $x \in C(\psi, b,(b / \gamma))$, from the assumption (A2), $f(t, x) \geq b / \delta$. Thus,

$$
\begin{aligned}
\psi(T x) & =\min _{0 \leq t \leq 1} \int_{0}^{1} G(t, s) \int_{0}^{s} f(\tau, x(\tau)) d \tau d s \\
& =\int_{0}^{1} G(1, s) \int_{0}^{s} f(\tau, x(\tau)) d \tau d s \\
& \geq \int_{0}^{1} s G(1, s) d s \times \frac{b}{\delta} \geq b,
\end{aligned}
$$

which ensures that condition $\left(\mathrm{H}_{1}\right)$ of Lemma 3 is satisfied. Finally, we show that condition $\left(\mathrm{H}_{3}\right)$ of Lemma 3 also holds. Suppose that $x \in C(\psi, b, c)$ with $\|T x\|>b / \gamma$,

$$
\psi(T x)=\min _{0 \leq t \leq 1} T x(t) \geq \gamma \times\|T x\|>\gamma \times \frac{b}{\gamma}=b .
$$

So, condition $\left(\mathrm{H}_{3}\right)$ of Lemma 3 is satisfied. Thus, an application of Lemma 3 implies that the nonresonant thirdorder boundary value problem (1) has at least three positive solutions $x_{1}, x_{2}$, and $x_{3}$ satisfying (31).

\section{Positive Solution for Resonant Problem}

In this section, the condition $\sum_{i=1}^{m-2} \beta_{i}=1$ is considered. Obviously, problem (1) is at resonance under this condition. The norm-type Leggett-Williams fixed point theorem will be used to establish the existence results of positive solution. We define the Banach spaces $X=Y=C[0,1]$ endowed with the maximum norm.

Define linear operator $L: \operatorname{dom} L \subset X \rightarrow Y, L x=$ $-x^{\prime \prime \prime}(t), t \in[0,1]$, where

$$
\begin{gathered}
\operatorname{dom} L=\left\{x \in X \mid x^{\prime \prime \prime} \in C[0,1], x^{\prime \prime}(0)=0,\right. \\
\left.x^{\prime}(0)=0, x(1)=\sum_{i=1}^{m-2} \beta_{i} x\left(\xi_{i}\right)\right\},
\end{gathered}
$$


and $N: X \rightarrow Y$ with

$$
(N x)(t)=f(t, x(t)), \quad t \in[0,1] .
$$

It is obvious that $\operatorname{Ker} L=\{x \in \operatorname{dom} L: x(t) \equiv c, t \in[0,1]\}$. Denote the function $G(s), s \in[0,1]$ as follows:

$$
\begin{array}{r}
G(s)=(1-s)^{2}-\sum_{i=k}^{m-1} \beta_{i}\left(\xi_{i}-s\right)^{2}, \quad \xi_{k-1} \leq s \leq \xi_{k} \\
k=1,2, \ldots, m-1 .
\end{array}
$$

Note that $G(s) \geq 0, s \in[0,1]$.

Denote the function $U(t, s)$ and positive number $\kappa$ as follows:

$$
\begin{aligned}
& U(t, s) \\
& =\left\{\begin{array}{l}
-\frac{1}{6} s^{3}+\frac{1}{2} s^{2}-\frac{1}{2} s+\frac{1}{6}+\frac{4 t^{3}+23}{24 \int_{0}^{1} G(s) d s} G(s), \\
0 \leq t \leq s \leq 1, \\
-\frac{1}{6} s^{3}-\frac{1}{2} s+s t-\frac{1}{2} t^{2}+\frac{1}{6}+\frac{4 t^{3}+23}{24 \int_{0}^{1} \frac{G(s) d s}{0 \leq s \leq t \leq 1,}} G(s), \\
\kappa:=\min \left\{1, \min _{s \in[0,1]} \frac{\int_{0}^{1} G(\tau) d \tau}{G(s)}, \min _{t, s \in[0,1]} \frac{1}{U(t, s)}\right\} .
\end{array}\right.
\end{aligned}
$$

Theorem 9. Assume that there exists positive constant $R \in$ $(0, \infty)$ such that $f:[0,1] \times[0, R] \rightarrow(-\infty,+\infty)$ is continuous and satisfies the following conditions:

(S1) $f(t, x) \geq-\kappa x$, for $(t, x) \in[0,1] \times[0, R]$,

(S2) $f(t, x)<0$ for $[t, x] \in[0,1] \times[(1-(\kappa / 2)) R, R]$,

(S3) there exists $r \in(0, R), t_{0} \in[0,1], a \in(0,1], M_{1}<M \in$ $(0,1)$ and continuous functions $g:[0,1] \rightarrow[0,+\infty)$, $h:(0, r] \rightarrow[0,+\infty)$ such that $f(t, x) \geq g(t) h(x)$, $[t, x] \in[0,1] \times(0, r]$ and $h(x) / x^{a}$ is nonincreasing on $(0, r]$ with

$$
\frac{h(r)}{r^{a}} \int_{0}^{1} U\left(t_{0}, s\right) g(s) d s \geq \frac{1-M}{M^{a}},
$$

then problem (1) with resonant condition (1.2) has at least one positive solution.

Proof. Firstly, we claim that

$$
\operatorname{Im} L=\left\{y \in Y \mid \int_{0}^{1} G(s) y(s) d s=0\right\} .
$$

In fact, for each $y \in\left\{y \in Y \mid \int_{0}^{1} G(s) y(s) d s=0\right\}$, we take

$$
x(t)=-\frac{1}{2} \int_{0}^{t}(t-s)^{2} y(s) d s .
$$

It is easy to check that $-x^{\prime \prime \prime}(t)=y(t), x^{\prime \prime}(0)=0, x^{\prime}(0)=0$, and $x(1)=\sum_{i=1}^{m-2} \beta_{i} x\left(\xi_{i}\right)$, which means $x(t) \in \operatorname{dom} L$. Thus,

$$
\left\{y \in Y \mid \int_{0}^{1} G(s) y(s) d s=0\right\} \subset \operatorname{Im} L .
$$

On the other hand, for each $y(t) \in \operatorname{Im} L$, there exists $x(t) \epsilon$ $\operatorname{dom} L$,

$$
\begin{gathered}
-x^{\prime \prime \prime}(t)=y(t), \quad x^{\prime \prime}(0)=0, \quad x^{\prime}(0)=0, \\
x(1)=\sum_{i=1}^{m-2} \beta_{i} x\left(\xi_{i}\right) .
\end{gathered}
$$

Integrating both sides on $[0, t]$, we have

$$
x(t)=-\frac{1}{2} \int_{0}^{t}(t-s)^{2} y(s) d s+\frac{1}{2} x^{\prime \prime}(0) t^{2}+x^{\prime}(0) t+x(0) .
$$

Considering condition $x^{\prime \prime}(0)=0, x^{\prime}(0)=0, x(1)=$ $\sum_{i=1}^{m-2} \beta_{i} x\left(\xi_{i}\right)$, and $\sum_{i=0}^{m-1} \beta_{i}=1$, we conclude that

$$
\int_{0}^{1}(1-s)^{2} y(s) d s-\sum_{i=0}^{m-1} \beta_{i} \int_{0}^{\xi_{i}}\left(\xi_{i}-s\right)^{2} y(s) d s=0,
$$

which equivalents to the conclusion that $\int_{0}^{1} G(s) y(s) d s=0$. So, we have

$$
\operatorname{Im} L \subset\left\{y \in Y \mid \int_{0}^{1} G(s) y(s) d s=0\right\} .
$$

Thus,

$$
\operatorname{Im} L=\left\{y \in Y \mid \int_{0}^{1} G(s) y(s) d s=0\right\} .
$$

Clearly, $\operatorname{dim} \operatorname{Ker} L=1$ and $\operatorname{Im} L$ are closed. Next, we see that $Y=Y_{1} \oplus \operatorname{Im} L$, where

$$
Y_{1}=\left\{y_{1} \mid y_{1}=\frac{1}{\int_{0}^{1} G(s) d s} \int_{0}^{1} G(s) y(s) d s, y \in Y\right\} .
$$

In fact, for each $y(t) \in Y$, we have

$$
\int_{0}^{1} G(s)\left[y(s)-y_{1}\right] d s=0 .
$$

This shows that $y-y_{1} \in \operatorname{Im} L$. Since $Y_{1} \cap \operatorname{Im} L=\{0\}$, we have $Y=Y_{1} \oplus \operatorname{Im} L$. Thus, $L$ is a Fredholm operator with index zero.

Then, define the projections $P: X \rightarrow X, Q: Y \rightarrow Y$ by

$$
P x=\int_{0}^{1} x(s) d s,
$$

$$
Q y=\frac{1}{\int_{0}^{1} G(s) d s} \int_{0}^{1} G(s) y(s) d s \text {. }
$$


Clearly, $\operatorname{Im} P=\operatorname{Ker} L, \operatorname{Ker} Q=\operatorname{Im} L$ and $\operatorname{Ker} P=\{x \in X$ : $\left.\int_{0}^{1} x(s) d s=0\right\}$. Note that for $y \in \operatorname{Im} L$, the inverse $K_{P}$ of $L_{P}$ is given by

$$
\left(K_{P}\right)(y)=\int_{0}^{1} k(t, s) y(s) d s
$$

where

$$
k(t, s)= \begin{cases}-\frac{1}{6} s^{3}+\frac{1}{2} s^{2}-\frac{1}{2} s+\frac{1}{6}, & 0 \leq t \leq s \leq 1, \\ -\frac{1}{6} s^{3}-\frac{1}{2} s+s t-\frac{1}{2} t^{2}+\frac{1}{6}, & 0 \leq s \leq t \leq 1 .\end{cases}
$$

In fact, it is easy to check that

$$
\begin{aligned}
L\left(K_{P}\right)(y) & =\left(-\int_{0}^{1} k(t, s) y(s) d s\right)^{\prime \prime \prime}=y(t), \\
K_{P}(L)(x)= & \int_{0}^{t}\left(-\frac{1}{6} s^{3}-\frac{1}{2} s+s t-\frac{1}{2} t^{2}+\frac{1}{6}\right)\left(-x^{\prime \prime \prime}(s)\right) d s \\
& +\int_{t}^{1}\left(-\frac{1}{6} s^{3}+\frac{1}{2} s^{2}-\frac{1}{2} s+\frac{1}{6}\right)\left(-x^{\prime \prime \prime}(s)\right) d s \\
= & x(t) .
\end{aligned}
$$

Considering that $f$ can be extended continuously on $[0,1] \times$ $(-\infty,+\infty)$; condition (C1) of Lemma 4 is fulfilled.

Define the cone of nonnegative functions $C$ and subsets of $X \Omega_{1}, \Omega_{2}$ by

$$
\begin{gathered}
C=\{x \in X: x(t) \geq 0, t \in[0,1]\}, \\
\Omega_{1}=\{x \in X: r>|x(t)|>M\|x\|, t \in[0,1]\}, \\
\Omega_{2}=\{x \in X:|x(t)|<R, t \in[0,1]\} .
\end{gathered}
$$

Clearly, $\Omega_{1}$ and $\Omega_{2}$ are bounded and open sets, furthermore

$$
\begin{array}{r}
\bar{\Omega}_{1}=\{x \in X: r \geq|x(t)| \geq M\|x\|, t \\
\qquad[0,1]\} \subset \Omega_{2}, \\
C \cap \bar{\Omega}_{2} \backslash \Omega_{1} \neq \varnothing .
\end{array}
$$

Let the isomorphism $J=I$ and $(\gamma x)(t)=|x(t)|$ for $x \in X$. Then, it is easy to check that $\gamma$ is a retraction and maps subsets of $\bar{\Omega}_{2}$ into bounded subsets of $C$, which means that condition (C3) of Lemma 4 is satisfied.

Next, we confirm that (C2) of Lemma 4 holds. For this purpose, suppose that there exists $x_{0} \in C \cap \partial \Omega_{2} \cap \operatorname{dom} L$ and $\lambda_{0} \in(0,1)$ such that $L x_{0}=\lambda_{0} N x_{0}$. Then,

$$
-x_{0}^{\prime \prime \prime}(t)=\lambda_{0} f\left(t, x_{0}\right)
$$

for all $t \in[0,1]$. Thus,

$$
\begin{gathered}
x_{0}^{\prime \prime}(t)=-\lambda_{0} \int_{0}^{t} f\left(s, x_{0}(s)\right) d s, \\
x_{0}^{\prime}(t)=-\lambda_{0} \int_{0}^{t}(t-s) f\left(s, x_{0}(s)\right) d s .
\end{gathered}
$$

Let $x_{0}\left(t_{0}\right)=\left\|x_{0}\right\|=R$. We verify that $t_{0} \neq 0$ and $t_{0} \neq 1$. The step is divided into three cases:

(1) we show that $t_{0} \neq 1$. Suppose, on the contrary, that $x_{0}(t)$ achieves maximum value $R$ only at $t=1$. Then, $x(1)=\sum_{i=1}^{m-2} \beta_{i} x_{0}\left(\xi_{i}\right)$ in combination with $\sum_{i=1}^{m-2} \beta_{i}=1$ yields that $\max _{1 \leq i \leq m-2} x_{0}\left(\xi_{i}\right) \geq R$, which is a contradiction,

(2) we show that $t_{0} \neq 0$. Suppose, on the contrary, that $x_{0}(t)$ achieves maximum value $R$ at $t=0$. Then,

$$
x_{0}^{\prime \prime \prime}(0)=-\lambda_{0} f(0, R)>0
$$

which together with the condition $x^{\prime \prime}(0)=x^{\prime}(0)=0$ yield that $x(t)$ is increasing near the point $t=0$. This contradicts to the fact that $x_{0}(t)$ achieves maximum value $R$ at $t=0$.

Thus, there exists $t_{0} \in(0,1)$ such that $x_{0}\left(t_{0}\right)=R=$ $\max _{0 \leq t \leq 1} x_{0}(t)$. We may choose $\eta<t_{0}$ nearest to $t_{0}$ with $x_{0}^{\prime \prime}(\eta)=0$. From the mean value theory, we claim that there exists $\xi \in\left(\eta, t_{0}\right)$ such that

$$
x_{0}(\eta)=x_{0}\left(t_{0}\right)-x_{0}^{\prime}(\xi)\left(t_{0}-\eta\right) .
$$

Here,

$$
\begin{aligned}
x_{0}^{\prime}(\xi) & =-\lambda_{0} \int_{0}^{\xi}(\xi-s) f\left(s, x_{0}\right) d s \\
& \leq \lambda_{0} \kappa \int_{0}^{\xi}(\xi-s) x_{0}(s) d s \\
& \leq \lambda_{0} \kappa R \int_{0}^{\xi}(\xi-s) d s=\frac{1}{2} \xi^{2} \lambda_{0} \kappa R .
\end{aligned}
$$

Thus,

$$
\begin{aligned}
x_{0}(\eta) & =x_{0}\left(t_{0}\right)-x_{0}^{\prime}(\xi)\left(t_{0}-\eta\right) \\
& \geq R-\frac{1}{2} \xi^{2} \lambda_{0} \kappa\left(t_{0}-\eta\right) R \\
& \geq\left(1-\frac{\kappa}{2}\right) R .
\end{aligned}
$$

Then,

$$
0 \geq x_{0}^{\prime \prime}\left(t_{0}\right)-x_{0}^{\prime \prime}(\eta)=-\lambda_{0} \int_{\eta}^{t_{0}} f\left(s, x_{0}(s)\right) d s,
$$

which contradict to condition (S2). Thus, (C2) holds.

Remark 10. The sign of third-order derivative of a function $y(t)$ at point $t_{0}$ cannot be confirmed even if $t_{0}$ is a maximal value of $y(t)$. Thus, the method in [29] is not applicable directly to problem (1). In our opinion, it is the key that the conditions (S2) in this paper are stronger than that in [29].

For $x \in \operatorname{Ker} L \cap \Omega_{2}$, define

$$
H(x, \lambda)=x-\lambda|x|-\frac{\lambda}{\int_{0}^{1} G(s) d s} \int_{0}^{1} G(s) f(s,|x|) d s,
$$


where $x=c \in \operatorname{Ker} L \cap \Omega_{2}$ and $\lambda \in[0,1]$. Suppose that $H(x, \lambda)=0$. In view of $(S 1)$, we obtain

$$
\begin{aligned}
c & =\lambda|c|+\frac{\lambda}{\int_{0}^{1} G(s) d s} \int_{0}^{1} G(s) f(s,|c|) d s \\
& \geq \lambda|c|-\frac{\lambda}{\int_{0}^{1} G(s) d s} \int_{0}^{1} G(s) \kappa|c| d s \\
& =\lambda|c|(1-\kappa) \geq 0 .
\end{aligned}
$$

Hence, $H(x, \lambda)=0$ implies $c \geq 0$. Furthermore, if $H(R, \lambda)=$ 0 , we get

$$
0 \leq R(1-\lambda) \int_{0}^{1} G(s) d s=\lambda \int_{0}^{1} G(s) f(s, R) d s,
$$

contradicting to (S2). Thus, $H(x, \lambda) \neq 0$ for $x \in \partial \Omega_{2}$ and $\lambda \epsilon$ $[0,1]$. Therefore,

$$
\begin{aligned}
d_{B}\left(H(x, 0), \operatorname{Ker} L \cap \Omega_{2}, 0\right) & =d_{B}\left(H(x, 1), \operatorname{Ker} L \cap \Omega_{2}, 0\right) \\
& =d_{B}\left(I, \operatorname{Ker} L \cap \Omega_{2}, 0\right)=1 .
\end{aligned}
$$

This ensures

$$
\begin{gathered}
d_{B}\left(\left.[I-(P+J Q N) \gamma]\right|_{\operatorname{Ker} L}, \operatorname{Ker} L \cap \Omega_{2}, 0\right) \\
=d_{B}\left(H(x, 1), \operatorname{Ker} L \cap \Omega_{2}, 0\right) \neq 0 .
\end{gathered}
$$

Let $x \in \bar{\Omega}_{2} \backslash \Omega_{1}$ and $t \in[0,1]$. From condition (S1), we see

$$
\begin{aligned}
\left(\Psi_{\gamma} x\right)(t)= & \int_{0}^{1}|x(t)| d t \\
& +\frac{1}{\int_{0}^{1} G(s) d s} \int_{0}^{1} G(s) f(s,|x(s)|) d s \\
& +\int_{0}^{1} k(t, s)\left[f(s,|x(s)|)-\frac{1}{\int_{0}^{1} G(s) d s}\right. \\
& \left.\quad \times \int_{0}^{1} G(\tau) f(\tau,|x(\tau)|) d \tau\right] d s \\
= & \int_{0}^{1}|x(t)| d t+\int_{0}^{1} U(t, s) f(s,|x(s)|) d s \\
\geq & \int_{0}^{1}|x(s)| d s-\kappa \int_{0}^{1} U(t, s)|x(s)| d s \\
= & \int_{0}^{1}(1-\kappa U(t, s))|x(s)| d s \geq 0 .
\end{aligned}
$$

Hence, $\Psi_{\gamma}\left(\bar{\Omega}_{2} \backslash \Omega_{1}\right) \subset C$. Moreover, for $x \in \partial \Omega_{2}$, we have

$$
\begin{aligned}
(P+J Q N) \gamma x= & \int_{0}^{1}|x(s)| d s \\
& +\frac{1}{\int_{0}^{1} G(s) d s} \int_{0}^{1} G(s) f(s,|x(s)|) d s \\
\geq & \int_{0}^{1}\left(1-\frac{\kappa}{\int_{0}^{1} G(s) d s} G(s)\right)|x(s)| d s \geq 0,
\end{aligned}
$$

which means $(P+J Q N) \gamma\left(\partial \Omega_{2}\right) \subset C$. These ensure that (C6) and (C7) of Lemma 4 hold.

At last, we confirm that (C5) is satisfied. Taking $u_{0}(t) \equiv 1$ on $[0,1]$, we see

$$
u_{0} \in C \backslash\{0\}, \quad C\left(u_{0}\right)=\{x \in C x(t)>0 \text { on }[0,1]\},
$$

and we can take $\sigma\left(u_{0}\right)=1$. Let $x \in C\left(u_{0}\right) \cap \partial \Omega_{1}$, we have

$$
\begin{gathered}
x(t)>0, \quad t \in[0,1], 0<\|x\| \leq r, \\
x(t) \geq M\|x\| \text { on }[0,1] .
\end{gathered}
$$

Therefore, in view of (S3), we obtain, for all $x \in C\left(u_{0}\right) \cap \partial \Omega_{1}$,

$$
\begin{aligned}
(\Psi x)\left(t_{0}\right) & =\int_{0}^{1} x(s) d s+\int_{0}^{1} U\left(t_{0}, s\right) f(s, x(s)) d s \\
& \geq M\|x\|+\int_{0}^{1} U\left(t_{0}, s\right) g(s) h(x(s)) d s \\
& =M\|x\|+\int_{0}^{1} U\left(t_{0}, s\right) g(s) \frac{h(x(s))}{x^{a}(s)} x^{a}(s) d s \\
& \geq M\|x\|+\frac{h(r)}{r^{a}} \int_{0}^{1} U\left(t_{0}, s\right) g(s) M^{a}\|x\|^{a} d s \\
& \geq M\|x\|+(1-M)\|x\|=\|x\| .
\end{aligned}
$$

So, $\|x\| \leq \sigma\left(u_{0}\right)\|\Psi x\|$, for all $x \in C\left(u_{0}\right) \cap \partial \Omega_{1}$, which means (C5) of Lemma 4 holds.

Thus, by Lemma 4 , we confirm that the equation $L x=$ $N x$ has a solution $x \in C \cap\left(\bar{\Omega}_{2} \backslash \Omega_{1}\right)$, which implies that nonlinear third-order multipoint boundary value problem (1) with resonance condition (1.2) has at least one positive solution.

\section{Examples}

In this section, we give two examples to illustrate the main results of the paper. First, we consider the nonresonant fourpoint boundary value problem

$$
\begin{gathered}
x^{\prime \prime \prime}(t)+f(t, x)=0, \quad t \in(0,1), \\
x^{\prime \prime}(0)=0, \quad x^{\prime}(0)=0, \\
x(1)=\frac{1}{2} x\left(\frac{1}{3}\right)+\frac{1}{3} x\left(\frac{2}{3}\right),
\end{gathered}
$$


where

$$
f(t, x)= \begin{cases}\frac{1}{100} e^{t}+\frac{x^{3}}{\pi}, & 0<x<6, \\ \frac{1}{100} e^{t}+\frac{216}{\pi}, & x \geq 6 .\end{cases}
$$

Here, $\beta_{1}=1 / 2, \beta_{2}=1 / 3, \xi_{1}=1 / 3, \xi_{2}=2 / 3$, and

$$
G(t, s)= \begin{cases}-s+\frac{11}{3}, & t \leq s, \quad 0 \leq s \leq \frac{1}{3}, \\ -t+\frac{11}{3}, & t \geq s, \quad 0 \leq s \leq \frac{1}{3}, \\ -4 s+\frac{14}{3}, & t \leq s, \frac{1}{3} \leq s \leq \frac{2}{3}, \\ -3 s-2 t+\frac{14}{3}, & t \geq s, \frac{1}{3} \leq s \leq \frac{2}{3}, \\ 6-6 s, & t \leq s, \frac{2}{3} \leq s \leq 1, \\ 6-t-5 s, & t \geq s, \frac{2}{3} \leq s \leq 1 .\end{cases}
$$

By a simply computation, we can get that

$$
m=\frac{143}{162}, \quad \delta=\frac{89}{162}, \quad \gamma=\frac{8}{11} .
$$

We choose $a=1, b=4$, and $c=162$. It is easy to check that

(1) $f(t, x)<143 / 162,[t, x] \in[0,1] \times[0,1]$,

(2) $f(t, x)>648 / 89,[t, x] \in[0,1] \times[4,11 / 2]$,

(3) $f(t, x)<143,[t, x] \in[0,1] \times[0,162]$.

Thus, all the conditions of Theorem 8 are satisfied. This ensures that problem (75) has at least three positive solutions $x_{1}, x_{2}$, and $x_{3}$ satisfying

$$
\begin{array}{ll}
\max _{0 \leq t \leq 1} x_{1} \leq 1, & \min _{0 \leq t \leq 1} x_{2}>4, \\
\max _{0 \leq t \leq 1} x_{3}>1, & \min _{0 \leq t \leq 1} x_{3}<4 .
\end{array}
$$

Next, we consider the resonant third-order four-point boundary value problem

$$
\begin{gathered}
x^{\prime \prime \prime}(t)+\left(-\frac{1}{2} t^{2}+\frac{1}{2} t+\frac{5}{16}\right)\left(x^{2}-4 x+\frac{11}{5}\right) \sqrt{x^{2}-6 x+10} \\
=0, \quad t \in[0,1], \\
x^{\prime \prime}(0)=0, \quad x^{\prime}(0)=0, \quad x(0)=x\left(\frac{2}{3}\right),
\end{gathered}
$$

where $\beta=1, \xi=2 / 3$ and

$$
\begin{aligned}
f(t, x)= & \left(-\frac{1}{2} t^{2}+\frac{1}{2} t+\frac{5}{16}\right) \\
& \times\left(x^{2}-4 x+\frac{11}{5}\right) \sqrt{x^{2}-6 x+10} .
\end{aligned}
$$

Here,

$$
G(s)= \begin{cases}\frac{5}{9}-\frac{2}{3} s, & 0 \leq s \leq \frac{2}{3} \\ (1-s)^{2}, & \frac{2}{3} \leq s \leq 1 .\end{cases}
$$

By a simple computation, we have

$$
\int_{0}^{1} G(s) d s=\frac{19}{81}, \quad \kappa=\frac{19}{45}, \quad \int_{0}^{1} U(0, s) d s=1 .
$$

Choose $R=1, r=1 / 4, t_{0}=0, a=1$, and $M=1 / 2$.

We take

$$
\begin{aligned}
& g(t)=-\frac{1}{2} t^{2}+\frac{1}{3} t+\frac{5}{16}, \quad t \in[0,1], \\
& h(x)=\sqrt{x^{2}-6 x+10}, \quad x \in\left[0, \frac{1}{4}\right] .
\end{aligned}
$$

Then,

$$
\begin{gathered}
\frac{7}{48} \leq g(t) \leq \frac{53}{144}<\frac{19}{45}, \quad t \in[0,1], \\
x^{2}-4 x+\frac{11}{5} \geq-x, \quad x \in[0,1] .
\end{gathered}
$$

It is easy to check that

(1) $f(t, x)>-(19 / 45) x$, for all $(t, x) \in[0,1] \times[0,1]$,

(2) $f(t, x)<0$, for all $(t, x) \in[0,1] \times[71 / 90,1]$,

(3) $f(t, x) \geq(101 / 80)\left(-(1 / 2) t^{2}+(1 / 3) t+(5 /\right.$ 16)) $\sqrt{x^{2}-6 x+10} \geq g(t) h(x),[t, x] \in[0,1] \times(0,1 / 4]$ and $h(x) / x=\sqrt{x^{2}-6 x+10} / x$

is nonincreasing on $(0,1 / 4]$ with

$$
\frac{h(r)}{r^{a}} \int_{0}^{1} U(0, s) g(s) d s>\frac{7 \sqrt{137}}{48}>1=\frac{1-M}{M^{a}} .
$$

Then, all conditions of Theorem 9 are satisfied. This ensures that the resonant problem has at least one solution, positive on $[0,1]$.

Remark 11. The established existence results of positive solutions for third-order boundary value problems in [2, 3, 5-12], for examples, are not applicable to the problem (75) or (5.2).

\section{Acknowledgments}

The paper is supported by the Natural Science Foundation of China (no. 11201109), the Natural Science Foundation of Anhui Educational Department (KJ2012Z335 and KJ2012B144), the Excellent Talents Foundation of University of Anhui Province (2012SQRL165), and the NSF of Hefei Normal University (2012Kj09). 


\section{References}

[1] M. Greguš, Third Order Linear Differential Equations, vol. 22 of Mathematics and its Applications, Reidel, Dordrecht, The Netherlands, 1987.

[2] D. Anderson, "Multiple positive solutions for a three-point boundary value problem," Mathematical and Computer Modelling, vol. 27, no. 6, pp. 49-57, 1998.

[3] D. J. Guo and V. Lakshmikantham, Nonlinear Problems in Abstract Cones, vol. 5, Academic Press, New York, NY, USA, 1988.

[4] A. P. Palamides and G. Smyrlis, "Positive solutions to a singular third-order three-point boundary value problem with an indefinitely signed Green's function," Nonlinear Analysis: Theory, Methods and Applications A, vol. 68, no. 7, pp. 2104-2118, 2008.

[5] L.-J. Guo, J.-P. Sun, and Y.-H. Zhao, "Existence of positive solutions for nonlinear third-order three-point boundary value problems," Nonlinear Analysis: Theory, Methods and Applications A, vol. 68, no. 10, pp. 3151-3158, 2008.

[6] B. Hopkins and N. Kosmatov, "Third-order boundary value problems with sign-changing solutions," Nonlinear Analysis: Theory, Methods and Applications A, vol. 67, no. 1, pp. 126-137, 2007.

[7] J. Chu and Z. Zhou, "Positive solutions for singular nonlinear third-order periodic boundary value problems," Nonlinear Analysis: Theory, Methods and Applications A, vol. 64, no. 7, pp. 1528-1542, 2006.

[8] J. R. Graef and L. Kong, "Positive solutions for third order semipositone boundary value problems," Applied Mathematics Letters, vol. 22, no. 8, pp. 1154-1160, 2009.

[9] X. Lin, Z. Du, and W. Liu, "Uniqueness and existence results for a third-order nonlinear multi-point boundary value problem," Applied Mathematics and Computation, vol. 205, no. 1, pp. 187196, 2008.

[10] L. Yang, W. Zhang, X. Liu, C. Shen, and H. Chen, "Positive solutions for a nonlinear third order multipoint boundary value problem," Pacific Journal of Mathematics, vol. 249, no. 1, pp. 177188, 2011.

[11] S. Li, "Positive solutions of nonlinear singular third-order two-point boundary value problem," Journal of Mathematical Analysis and Applications, vol. 323, no. 1, pp. 413-425, 2006.

[12] Q. Yao, "Solution and positive solution for a semilinear thirdorder two-point boundary value problem," Applied Mathematics Letters, vol. 17, no. 10, pp. 1171-1175, 2004.

[13] W. Feng and J. Webb, "Solvability of three point boundary value problems at resonance," Nonlinear Analysis: Theory, Methods and Applications A, vol. 30, no. 6, pp. 3227-3238, 1997.

[14] I. Rachůnková, "On the existence of two solutions of the four-point problem," Journal of Mathematical Analysis and Applications, vol. 193, no. 1, pp. 245-254, 1995.

[15] I. Rachůnková, "Upper and lower solutions and topological degree," Journal of Mathematical Analysis and Applications, vol. 234, no. 1, pp. 311-327, 1999.

[16] C.-C. Kuo, "Solvability of a nonlinear two-point boundary value problem at resonance," Journal of Differential Equations, vol. 140, no. 1, pp. 1-9, 1997.

[17] B. Liu, "Solvability of multi-point boundary value problem at resonance. II," Applied Mathematics and Computation, vol. 136, no. 2-3, pp. 353-377, 2003.

[18] B. Liu and J. Yu, "Solvability of multi-point boundary value problem at resonance. III," Applied Mathematics and Computation, vol. 129, no. 1, pp. 119-143, 2002.
[19] B. Liu, "Solvability of multi-point boundary value problem at resonance. IV," Applied Mathematics and Computation, vol. 143, no. 2-3, pp. 275-299, 2003.

[20] Z. Bai, W. Li, and W. Ge, "Existence and multiplicity of solutions for four-point boundary value problems at resonance," Nonlinear Analysis: Theory, Methods and Applications A, vol. 60, no. 6, pp. 1151-1162, 2005.

[21] Z. Du, X. Lin, and W. Ge, "Some higher-order multi-point boundary value problem at resonance," Journal of Computational and Applied Mathematics, vol. 177, no. 1, pp. 55-65, 2005.

[22] X. Lin, Z. Du, and W. Ge, "Solvability of multipoint boundary value problems at resonance for higher-order ordinary differential equations," Computers and Mathematics with Applications, vol. 49, no. 1, pp. 1-11, 2005.

[23] S. Lu and W. Ge, "On the existence of $m$-point boundary value problem at resonance for higher order differential equation," Journal of Mathematical Analysis and Applications, vol. 287, no. 2, pp. 522-539, 2003.

[24] Y. Liu and W. Ge, "Solvability of nonlocal boundary value problems for ordinary differential equations of higher order," Nonlinear Analysis: Theory, Methods and Applications A, vol. 57, no. 3, pp. 435-458, 2004.

[25] Z. Du, X. Lin, and W. Ge, "On a third-order multi-point boundary value problem at resonance," Journal of Mathematical Analysis and Applications, vol. 302, no. 1, pp. 217-229, 2005.

[26] C. Bai and J. Fang, "Existence of positive solutions for threepoint boundary value problems at resonance," Journal of Mathematical Analysis and Applications, vol. 291, no. 2, pp. 538-549, 2004.

[27] D. O'Regan and M. Zima, "Leggett-Williams norm-type theorems for coincidences," Archiv der Mathematik, vol. 87, no. 3, pp. 233-244, 2006.

[28] D. O'Regan and M. Zima, "Leggett-Williams theorems for coincidences of multivalued operators," Nonlinear Analysis: Theory, Methods and Applications A, vol. 68, no. 10, pp. 28792888, 2008.

[29] G. Infante and M. Zima, "Positive solutions of multi-point boundary value problems at resonance," Nonlinear Analysis: Theory, Methods and Applications A, vol. 69, no. 8, pp. 24582465, 2008.

[30] L. Yang and C. Shen, "On the existence of positive solution for a kind of multi-point boundary value problem at resonance," Nonlinear Analysis: Theory, Methods and Applications A, vol. 72, no. 11, pp. 4211-4220, 2010.

[31] D. Franco, G. Infante, and M. Zima, "Second order nonlocal boundary value problems at resonance," Mathematische Nachrichten, vol. 284, no. 7, pp. 875-884, 2011.

[32] R. W. Leggett and L. R. Williams, "Multiple positive fixed points of nonlinear operators on ordered Banach spaces," Indiana University Mathematics Journal, vol. 28, no. 4, pp. 673-688, 1979. 


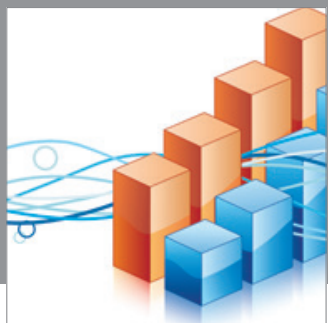

Advances in

Operations Research

mansans

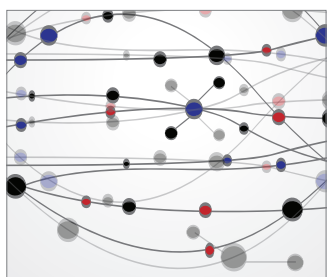

The Scientific World Journal
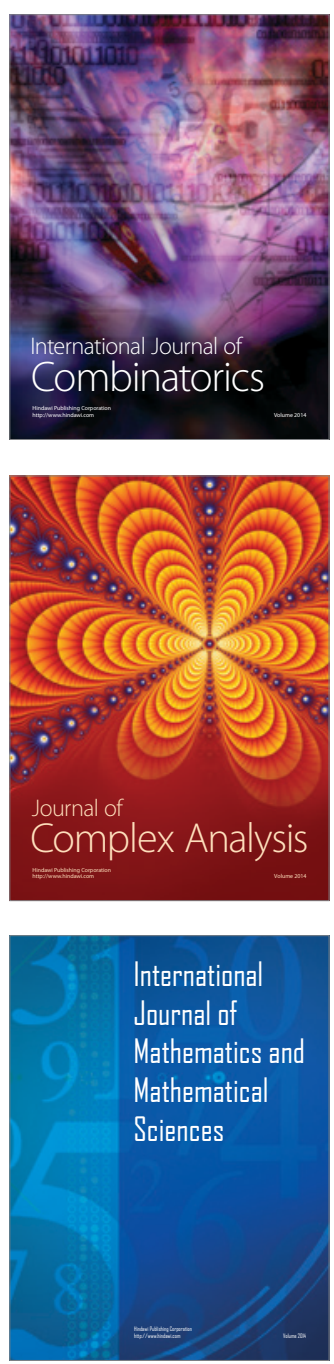
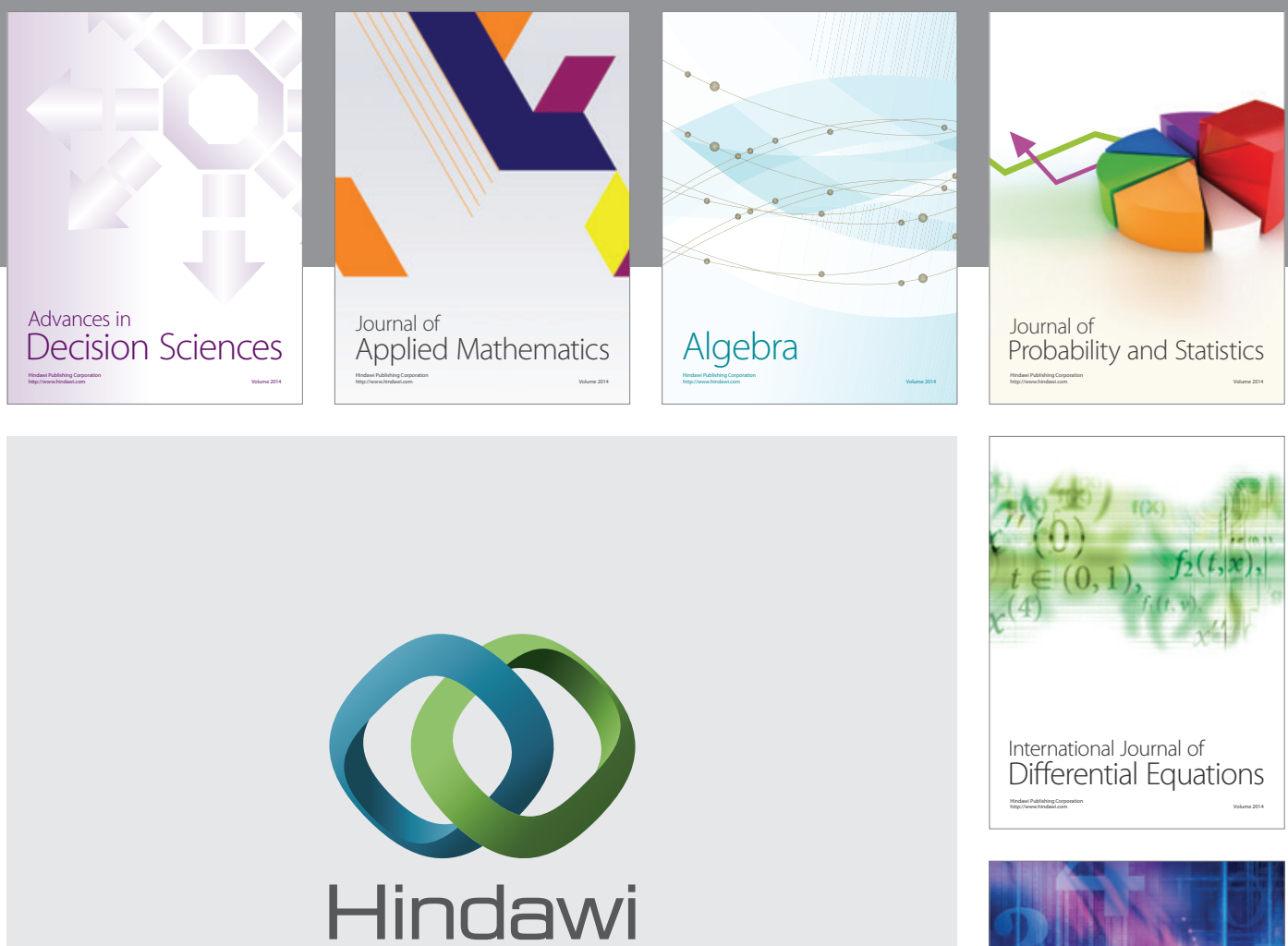

Submit your manuscripts at http://www.hindawi.com
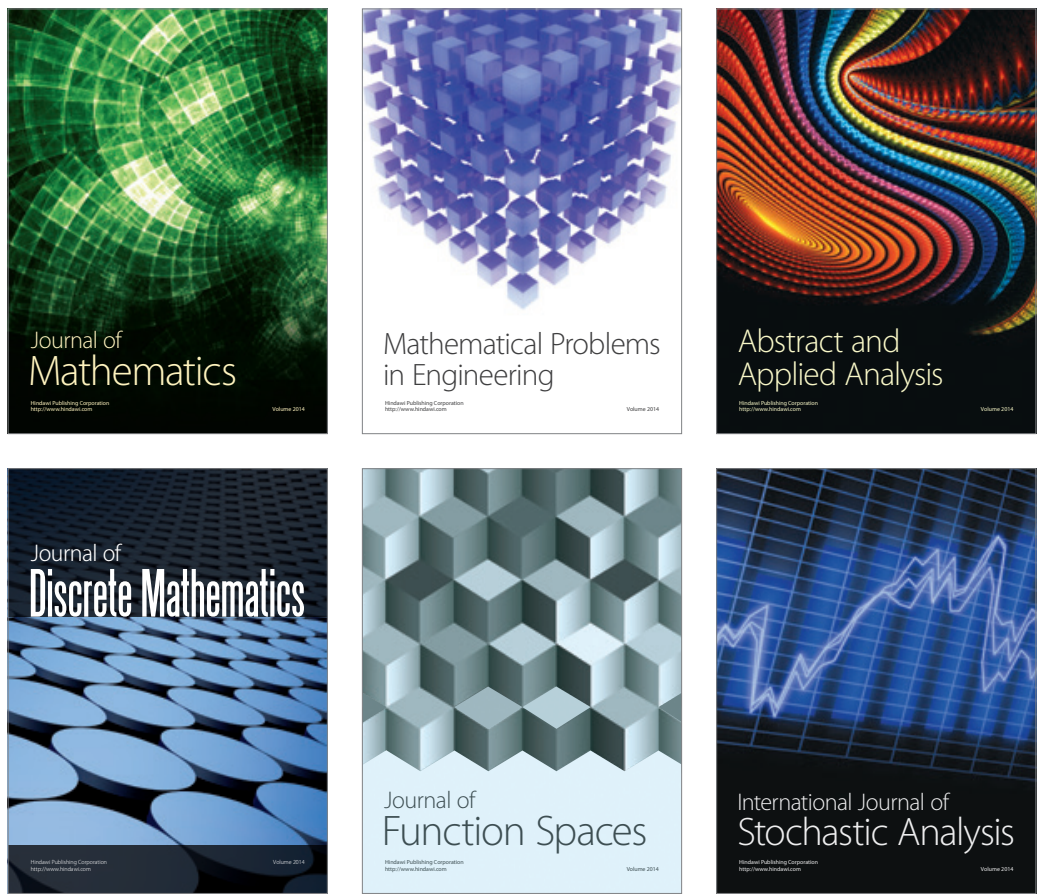

Journal of

Function Spaces

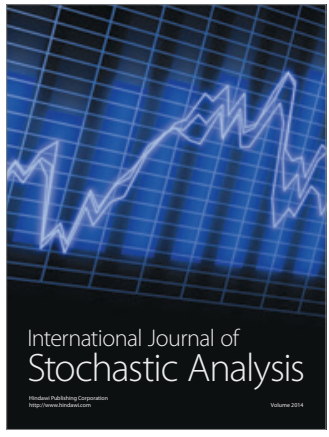

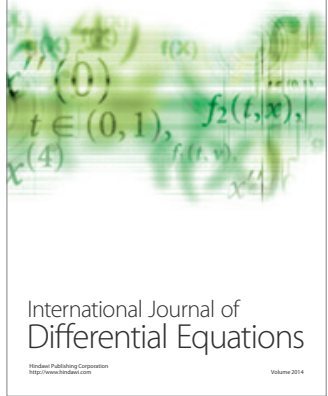
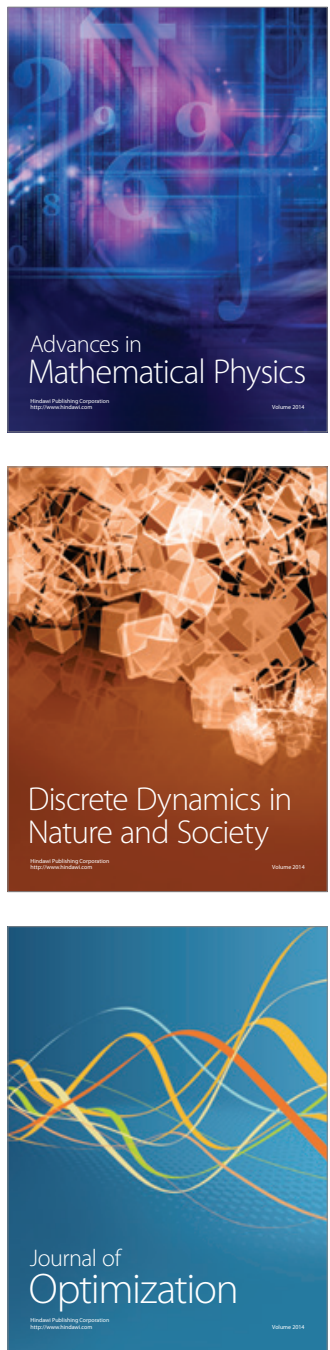